\title{
Effect of aging on the tendon structure and tendon-associated gene expression in mouse foot flexor tendon
}

\author{
YOICHI SUGIYAMA ${ }^{1}$, KIYOHITO NAITO ${ }^{1}$, KENJI GOTO ${ }^{1}$, YUKO KOJIMA ${ }^{2}$, \\ ATSUSHI FURUHATA ${ }^{2}$, MAMORU IGARASHI ${ }^{3}$, ISAO NAGAOKA ${ }^{3}$ and KAZUO KANEKO ${ }^{1}$ \\ ${ }^{1}$ Department of Medicine for Motor Organs; ${ }^{2}$ Laboratory of Morphology and Image Analysis, \\ Research Support Center; ${ }^{3}$ Department of Host Defense and Biochemical Research, \\ Juntendo University Graduate School of Medicine, Tokyo 113-8421, Japan
}

Received December 13, 2018; Accepted March 7, 2019

DOI: $10.3892 /$ br.2019.1200

\begin{abstract}
To evaluate the biological changes in tendons during the aging process, the present study examined the effect of aging on the tendon structure, distribution of collagen types I and III, and expression of tendon-associated genes, using flexor tendons in a mouse model. Histological assessment of the tendon structure and distribution of collagen types I and III were performed, and the expression of tendon-associated genes was evaluated in flexor digitorium longus tendons of young (8 weeks) and aged (78 weeks) female C57BL/6 mice. The results indicated that the Soslowsky score, based on the analysis of cellularity, fibroblastic changes, and collagen fiber orientation and disruption, was significantly increased, or worsened, in the tendons of the aged group compared with those in the young group. Furthermore, in the aged group, the distribution of type I collagen was decreased and the distribution of type III collagen was relatively increased compared with the young group. Finally, the mRNA expression levels of collagen (type I and type III) and tenogenic markers (Mohawk homeobox, tenomodulin and scleraxis BHLH transcription factor) were significantly decreased in the aged group compared with the young group. The present observations demonstrated that the structure of the tendons, distribution of types I and III collagen and the expression of tendon-associated genes were modulated by aging in the flexor tendon, and that these changes may contribute to the degeneration of tendons in tendinopathy.
\end{abstract}

Correspondence to: Professor Isao Nagaoka, Department of Host Defense and Biochemical Research, Juntendo University Graduate School of Medicine, 2-1-1 Hongo, Bunkyo-ku, Tokyo 113-8421, Japan

E-mail: nagaokai@juntendo.ac.jp

Key words: tendon, collagen, aging, tendinopathy, tendon-associated gene

\section{Introduction}

Aging is a complex and varied process that involves the accumulation of numerous molecular changes and resulting pathological alterations to normal physiological function (1). These aging-associated changes involve various tissues, for example skin, bones and muscles, and have attracted much attention in the investigation of the mechanisms involved in the pathophysiology of aging-associated disorders, including age-induced osteoporosis (2-5). Tendons are fibrous tissues that connect muscle to bone, and are able to resist high levels of force. Their primary function is to transfer muscle-generated force to the skeleton, facilitating movement around a joint. Notably, there are clinical data demonstrating the association between aging and an increased incidence of tendon rupture (6); however, to the best of our knowledge, basic studies, animal models and clinical studies using patients examining aging-associated tendon degeneration have not been performed.

A healthy tendon is a fibrous type of tissue with a highly organized type I collagen-based extracellular matrix, and a minimal number of cells and neurovascular structures (7). As tendons mature postnatally, tenocytes slowly proliferate to become largely quiescent, resulting in an extremely slow tissue turnover rate but an absence of atrophy with aging $(1,2)$. A previous study suggested that one of the key characteristics of chronic tendinopathy is the degradation of the extracellular matrix collagen (8). Other data indicated that a marked loss of bridging collagen in the extracellular matrix was observed in repaired tendons in an aged mouse mode (9). These observations suggest that the decreased proliferation of tenocytes and increased matrix degeneration lead to impaired tendon healing with aging (9). However, the basic mechanisms underlying the pathological changes of tendons with aging are not well understood. The majority of previous studies have investigated the mechanical features and the healing process of aged tendons; to the best of our knowledge, only one study has focused on the effect of aging on the biological features of tendons (10).

In the present study, to evaluate the biological and pathological changes of tendons with aging, the effect of aging on the tendon structure, distribution of collagen types I and III and expression of tendon-associated genes was examined using flexor tendons in a mouse model. 


\section{Materials and methods}

Animal model. Female C57BL/6 mice $(n=26)$ were used in the present study. Mice were obtained from Japan SLC, Inc., (Shizuoka, Japan). Animals were housed in groups, with up to 5 animals/cage in a pathogen-free environment at $22 \pm 2^{\circ} \mathrm{C}$ with 40-60\% humidity under a $12 \mathrm{~h}$ light-dark cycle with ad libitum access to food and water. The effect of aging on the structure and gene expression in the tendons was assessed at 8 weeks (young group) and 78 weeks (aged group); 5 and 8 mice (young or aged) were for employed for structural and gene expression analyses, respectively. The present study was approved by the Animal Care Committee of Juntendo University, Tokyo, Japan (registration no. 1309; approval no. 300052).

Histological assessment of tendon structure. Following euthanasia, flexor digitorium longus (FDL) tendons of the right hind paws (including 5 digital tendons and a bunched portion of the tendons) were harvested under a light microscope (Zeiss Axioskop2; magnification, $\mathrm{x} 40$ ) for histological analysis. Then, tendons were fixed in $4 \%$ paraformaldehyde at room temperature for $72 \mathrm{~h}$, and processed and embedded in paraffin. From the bunched portion of tendons, $3 \mu \mathrm{m}$ sagittal sections were prepared and stained with $0.1 \%$ hematoxylin for $5 \mathrm{~min}$ and $1 \%$ eosin for $2 \mathrm{~min}$ at room temperature ( 5 sections/mouse). In addition, $3 \mu \mathrm{m}$ axial sections were prepared from the bunched portion of tendons, and stained with $0.1 \%$ Picrosirius Red (Waldeck GmbH \& Co. KG, Münster, Germany) for 90 min at room temperature ( 8 sections/mouse).

Soslowsky score. The Soslowsky score is a histological scoring system, which is used to investigate tendinopathies and tendon repair (11). The total score may range from 0 (normal tendon)-12 (most severe degeneration detectable). Sections were stained with $0.1 \%$ hematoxylin for $5 \mathrm{~min}$ and $1 \%$ eosin for $2 \mathrm{~min}$ at room temperature to assess cellularity (score 0-3), fibroblastic changes (score 0-3), and collagen fiber orientation (score 0-3) and disruption (score 0-3). The analysis was accomplished using a semiquantitative method by evaluating scores (0-3) of each component (cellularity, fibroblastic change, collagen fiber orientation and disruption) (12). The slides were randomly selected and evaluated by two blinded observers under a light microscope (magnification, $\mathrm{x} 20$ ). The total score of each mouse (5 sections/mouse) was averaged, and compared between the young and aged groups (5 mice/group).

Picrosirius red staining for collagen. Picrosirius red staining is utilized for evaluating the differential distribution of the structurally distinct collagen types I and III (13-15). Picrosirius red-stained sections were illuminated with polarized light, allowing the visualization of collagen fiber organization; type I collagen appears red, while type III collagen appears yellow/green (16). Digital images of all specimens were captured and analyzed with a custom-built spectral template (red or yellow/green) using the Nuance multispectral imaging system (Nuance 3.0.2; PerkinElmer, Inc., Waltham, MA, USA). The total number of pixels of red and yellow/green in the specimen was obtained, and the percentage of red (type I collagen) or yellow/green (type III collagen) staining was calculated. This proportion was averaged in each mouse
(8 sections/mouse) and compared between young and aged groups (5 mice/group).

Reverse transcription quantitative polymerase chain reaction $(R T-q P C R)$. FDL tendons of the right and left hind paws were harvested from each mice ( 8 mice/young or aged group) using microscopy.

Total RNA was isolated from flexor tendons (2 tendons/mouse) with an RNeasy Fibrous Tissue Mini kit (Qiagen, Inc., Valencia, CA, USA). cDNA was synthesized using qPCR RT Master Mix (Toyobo Life Science, Osaka, Japan) at $37^{\circ} \mathrm{C}$ for $15 \mathrm{~min}$ and at $50^{\circ} \mathrm{C}$ for $5 \mathrm{~min}$. qPCR was performed using an ABI Prism 7500 sequence detection system (Applied Biosystems; Thermo Fisher Scientific, Inc., Waltham, MA, USA) with the SYBR-Green PCR Master Mix (Toyobo Life Science, Osaka, Japan), according to the manufacturer's protocol. The detector was programmed with the following PCR thermocycler conditions: Initial denaturation at $95^{\circ} \mathrm{C}$ for $1 \mathrm{~min}, 40$ cycles of $15 \mathrm{sec}$ denaturation at $95^{\circ} \mathrm{C}$ and $1 \mathrm{~min}$ amplification (annealing and elongation) at $60^{\circ} \mathrm{C}$. All reactions were performed in triplicate and normalized to the level of the housekeeping gene glyceraldehyde-3-phosphate dehydrogenase (GAPDH). The relative differences in the PCR results were calculated using the comparative cycle threshold method (17). The following primer sets were used: Type I collagen forward, 5'-GCTCCTCTTAGGGGC CACT-3'; type I collagen reverse, 5'-CCACGTCTCACCATTGGGG-3'; type III collagen forward, 5'-ACGTAGATGAATTGGGATG CAG-3'; type III collagen reverse, 5'-GGGTTGGGGCAGTC TAGTG-3'; tenomodulin forward, 5'-TGTACTGGATCAATC CCACTCT-3'; tenomodulin reverse, 5'-GCTCATTCTGGTC AATCCCCT-3'; scleraxis BHLH transcription factor forward, 5'-CACCCAGCCCAAACAGATCTG CA-3'; scleraxis BHLH transcription factor reverse, 5'-AGTGGCATCACCTCTTGG CTGCT-3'; Mohawk homeobox forward, 5'-CACCGTGACA ACCCGTACC-3'; Mohawk homeobox reverse, 5'-GCACTA GCGTCATCTGCGAG-3'; GAPDH forward, 5'-ATGGCCTT CCGTGTTTCCTAC-3'; and GAPDH reverse, 5'-TGATGT CATCATACTTGGCAGG-3'.

Statistical analysis. Values are expressed as the means \pm standard deviation. Statistical analysis was performed by comparing values between the young and aged groups with a Mann-Whitney U test using GraphPad Prism 7 (GraphPad Software, Inc., La Jolla, CA, USA). P $<0.05$ was considered to indicate a statistically significant difference.

\section{Results}

Histological assessment of tendon structure. Firstly, the structural changes in the FDL tendons of the hind paws in the young and aged groups was evaluated by hematoxylin and eosin staining of tissue sections. Normal collagen is stained pink-red by hematoxylin and eosin, although the intensity of the staining is decreased and appears paler with the degeneration of collagen (18). In the present study, the collagen fibers in the sections of the young group were stained pink-red by hematoxylin and eosin (Fig. 1A). However, the staining intensity was decreased in the aged group (Fig. 1B). Furthermore, the cell number, or cellularity, was observed to be decreased and 


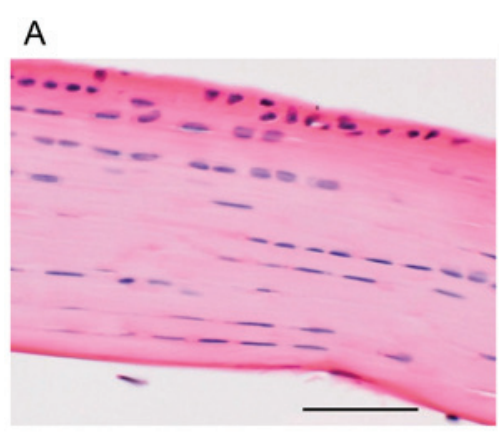

B
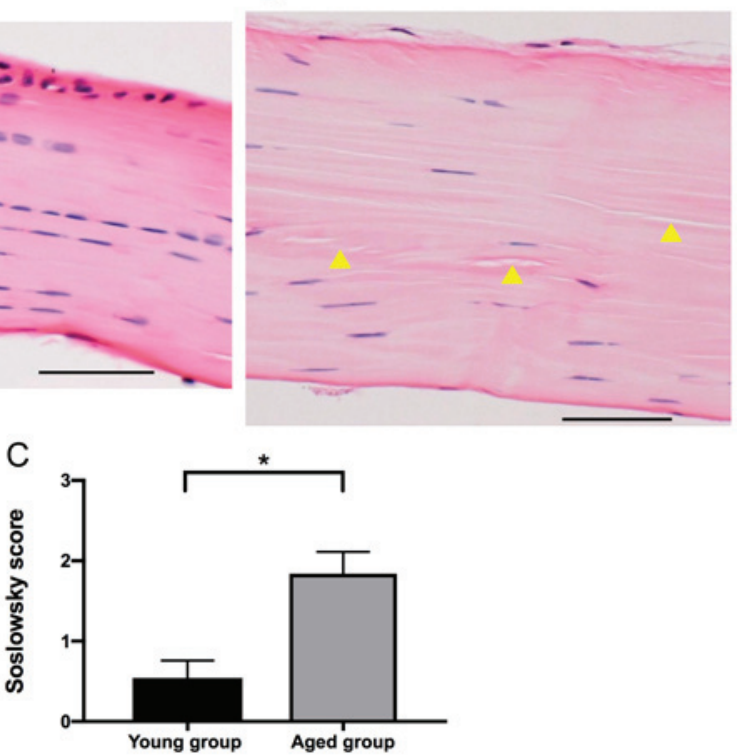

Figure 1. Histological assessment of tendon structure and Soslowsky score. (A and B) Sections of flexor digitorium longus tendons in the (A) young and (B) aged groups were stained by hematoxylin and eosin. Nuclei of tenocytes are stained in violet. Yellow arrowheads indicate the altered and disrupted orientation of collagen fibers in the aged group. Scale bar=50 $\mu \mathrm{m}$. (C) The Soslowsky score was calculated, based on the cellularity, fibroblastic changes, and collagen fiber orientation and disruption. Data values are expressed as the means \pm standard deviation. ${ }^{*} \mathrm{P}<0.05$.

A

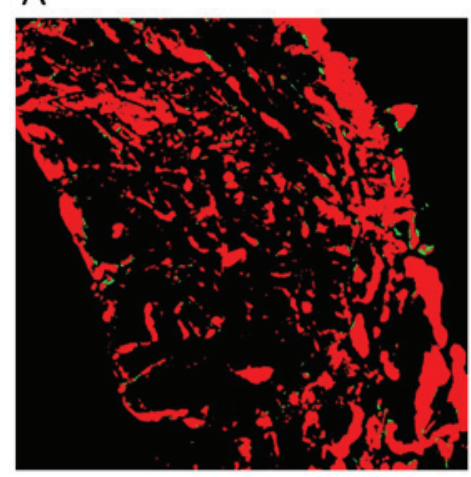

C

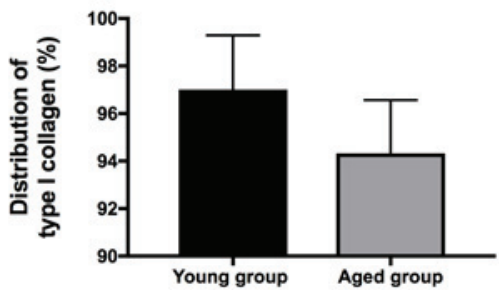

B

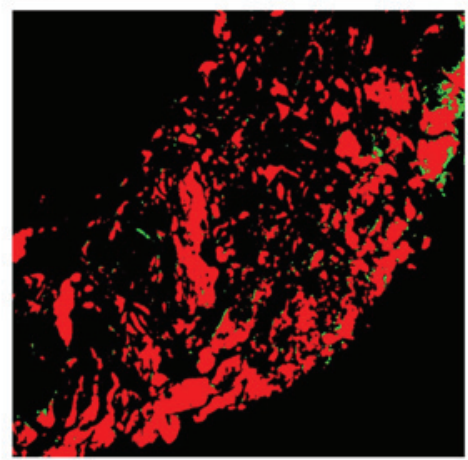

D

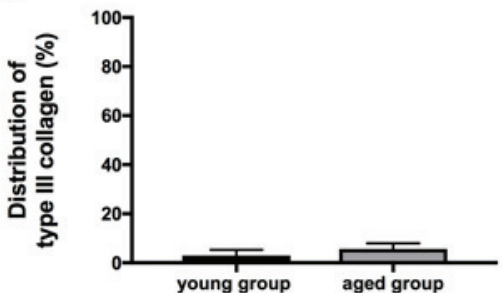

Figure 2. Evaluation of the differential distribution of collagen types I and III. (A and B) Sections of flexor digitorium longus tendons in the (A) young and (B) aged groups were stained by picrosirius red, which allowed visualization of type I collagen (red) and type III collagen (yellow/green). Magnification, x40. (C and D) The total number of red and yellow/green pixels in these images were analyzed with the Nuance multispectral imaging system, and the distribution of (C) type I collagen (red/total pixels) and (D) type III collagen (yellow and green/total pixels) were calculated, and expressed as proportions. Values are expressed as the means \pm standard deviation of the young $(n=5)$ and aged $(n=5)$ groups.

the shape of nuclei became elongated, representing fibroblastic changes, in the aged group compared with the young group. In addition, the alignment, or orientation, of the collagen fibers was altered and disrupted in the aged group (Fig. 1B). Based on the results from the analysis of cellularity, fibroblastic changes, and collagen fiber orientation and disruption, the Soslowsky score was calculated; the score was significantly increased, or worsened, in the tendons of the aged group compared with that in young group $(\mathrm{P}<0.05$; Fig. $1 \mathrm{C})$.

Evaluation of the differential distribution of collagen types I and III. Next, the differential distribution of collagen types I and III in the tendons of the young and aged groups was evaluated. Type I collagen is the predominant collagen 
A

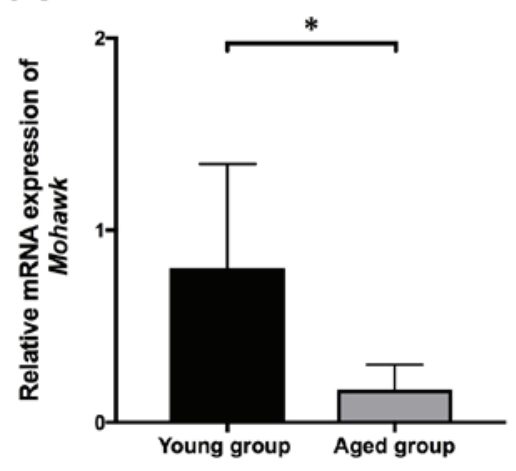

D

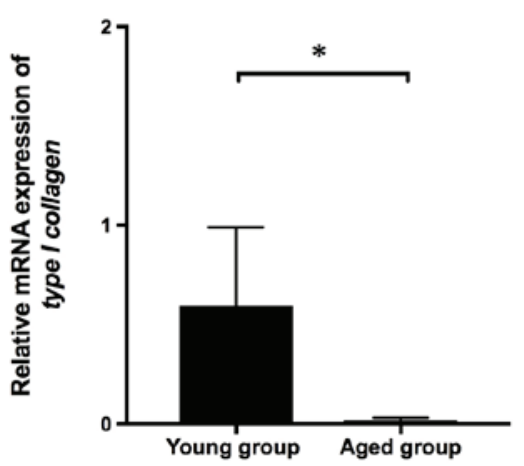

B

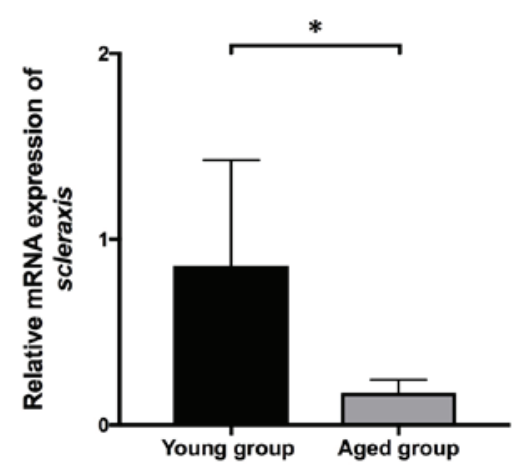

C

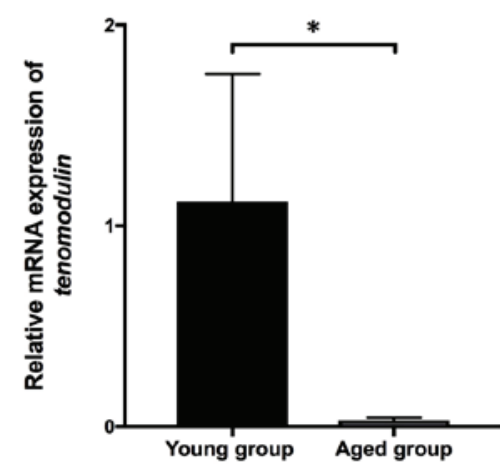

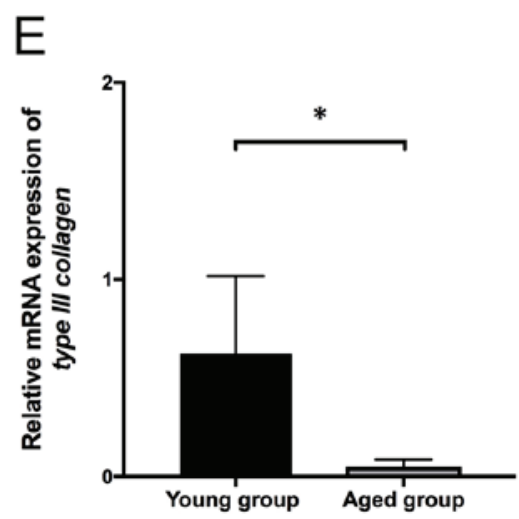

Figure 3. mRNA expression of tendon-associated genes. mRNA expression of (A) Mohawk, (B) scleraxis, (C) tenomodulin, (D) type I collagen and (E) type III collagen in flexor digitorium longus tendons of the young and aged groups was evaluated by reverse transcription quantitative polymerase chain reaction. The mRNA expression levels are presented as relative quantities in comparison with that of GAPDH mRNA. Values are expressed as the means \pm standard deviation, and comparisons were made between the young $(n=8)$ and aged $(n=8)$ groups. * $\mathrm{P}<0.05$. Mohawk, Mohawk homeobox; scleraxis, scleraxis BHLH transcription factor.

in tendons, comprising 90-95\% of the total collagen content, and is localized in the tendon parenchyma $(19,20)$. By contrast, type III collagen is the second most abundant type of collagen in the tendon, comprising up to 5-10\% of the collagen content. In normal tendons, type III collagen is primarily located in the endotenon, the fine connective tissue between the strands in a tendon, and the epitenon, which is a sheath around the tendons (21). Therefore, the present study assessed the effect of aging on the differential distribution of collagen type I and III by using picrosirius red staining, which provides a visualization of type I collagen (red) and type III collagen (yellow/green) distribution in tissues. In the picrosirius red-stained sections of the young and aged groups, type I collagen (red) was localized in the tendon parenchyma, whereas type III collagen (yellow/green) was localized around the bundle of type I collagen (red) (Fig. 2A and B). Of note, relative staining of type I collagen (red) to type III collagen (yellow/green) was markedly decreased in the aged group compared with that in the young group (Fig. 2A and B). Furthermore, the total number of red and yellow/green pixels in the images were obtained and analyzed with a Nuance multispectral imaging system. As demonstrated in Fig. 2C and D, the percentage of type I collagen was decreased $(\mathrm{P}=0.15)$ and the percentage of type III collagen was relatively increased $(\mathrm{P}=0.15)$ in the aged group compared with the young group, although no statistical significance between the two groups was observed.
Expression of tendon-associated genes. Finally, the effect of aging on the mRNA expression of type I and type III collagen and tendon-associated genes was evaluated. Mohawk homeobox, a transcription factor, serves a critical role in tendon differentiation by regulating type I collagen production in tendon cells (22); scleraxis BHLH transcription factor, a transcription factor, has been demonstrated to trigger tendon differentiation in stem cells and improve repair of tendon injury in animal models (23). Tenomodulin is a type II transmembrane glycoprotein that is highly expressed in tendons, and functions as a regulator of tenocyte proliferation and collagen fibril maturation (24). Notably, mRNA expression levels of type I and type III collagen and tenogenic markers (Mohawk homeobox, tenomodulin and scleraxis BHLH transcription factor) were significantly decreased in the aged group compared with the young group $(\mathrm{P}<0.05$; Fig. 3$)$.

\section{Discussion}

The aim of the present study was to evaluate the aging-associated changes of tendons using young and aged mice, and to clarify the basic mechanism for the increased incidence of tendinopathy associated with aging. Previous studies using a flexor tendon injury model primarily focused on the morphological changes at the site of repair and the cellular source contributing to tendon healing $(9,25)$. However, to the best of our knowledge, the effects of aging on the histological and biochemical 
features of tendons have not been explored thoroughly. In the present study, histological assessment of tendon structure was performed and the expression levels of tendon-associated genes in young and aged mice were examined. The results indicated that the Soslowsky score, based on the analysis of cellularity, fibroblastic changes, and collagen fiber orientation and disruption, was significantly increased, or worsened, in the tendons of the aged group compared with those in the young group. Furthermore, in the aged group, the distribution of type I collagen was decreased and the distribution of type III collagen was relatively increased compared with the young group. Finally, the mRNA expression levels of collagen (type I and type III) and tenogenic markers (Mohawk homeobox, tenomodulin and scleraxis BHLH transcription factor) were significantly decreased in the aged group compared with the young group. Therefore, the data from the present study demonstrated that aging modulates the structure of tendons, distribution of types I and III collagen and the expression of tendon-associated genes.

It has been demonstrated that the number of tenocytes decreases during the aging process, and that the remaining cells exhibit an altered morphology, with rounder cells in the younger tendons being replaced by a thinner, more elongated phenotype in aged tenocytes, which is associated with a lower metabolic activity, including decreased collagen synthesis $(9,26)$. The decrease in cellularity may be associated with a decrease in the size of the pool of tendon/stem progenitor cells (27-29). Consistent with these data, the present study revealed that the staining intensity, and potentially the protein content, based on the result of western blot analysis (as described below), of the collagen fibers was decreased, the tenocyte number was decreased, and the shape of nuclei became elongated in the aged group compared with the young group. Notably, preliminary western blot analysis experiments in the present study confirmed that the content of type I collagen, the predominant collagen in tendons, was decreased in the aged group compared with the young group (data not shown). However, type III collagen was not detected by western blot analysis, due to its low expression level. As the total amount of collagen was observed to be markedly decreased in the tendons of the aged group by hematoxylin and eosin staining, we hypothesized that the protein content of type III was also decreased in the aged tendons, reflecting the decreased mRNA expression of type III collagen; however, the levels of type III collagen were demonstrated to be relatively increased from $3.0 \%$ in the young group to $5.6 \%$ in the aged group by picrosirius red staining. Furthermore, it has been suggested that levels of elasticity and tension decrease in skin when the levels of type III collagen decrease (30). However, in the present study, the distribution of type I and type III collagen was only examined in the tendon, but not in other organs and tissues. The changes in type I and type III collagen in other organs and tissues with aging should be evaluated in future studies.

Notably, the histopathological scores between young and aged tendons were compared using the Soslowsky score system, which may be used for the evaluation of tendinopathies and tendon repair, based on the cellularity, fibroblastic changes, and collagen fiber orientation and disruption. As a result, the score was significantly increased, or worsened, in the tendons of the aged group compared with those in the young group.
This observation supports previous data that the cellularity and tendon matrix in tendons may decrease with age, which results in the increased incidence of tendinopathy (9).

Furthermore, it has been revealed that mild and moderate matrix degeneration in tendinopathy characteristically exhibits a decrease in collagen type I but an increase in collagen type III (31). Notably, the picrosirius red staining performed in the present study demonstrated that in the aged group, the distribution of type I collagen was decreased but the distribution of type III collagen was relatively increased compared with the young group. This change in the distribution of collagen (types I and III) may contribute to the development of age-associated tendinopathy.

Accumulating evidence has suggested that scleraxis BHLH transcription factor-expressing tenogenic cells may participate in the regenerative repair of tendons to promote the synthesis of collagen fibers with an aligned orientation, which have improved tensile strength (32). Tenomodulin is a regulator of tenocyte proliferation and involved in collagen fibril maturation (24). In addition, it has been demonstrated clearly that tenomodulin is necessary for the maintenance and repair of tendons (23). In addition, Mohawk homeobox serves a critical role in the regulation of type I collagen production in tendon cells (22). The present study revealed that the mRNA expression levels of tenogenic markers Mohawk homeobox, tenomodulin and scleraxis BHLH transcription factor were significantly decreased in the aged group compared with those in the young group. Furthermore, mRNA expression of collagen (types I and III) was significantly decreased in the aged group compared with the young group. Taken together, these observations suggest that the synthesis and maturation of collagen and tenocyte proliferation are suppressed in the flexor tendon with aging, and these changes are likely to contribute to the degeneration of tendons in tendinopathy.

Stenosing tenosynovitis is a common tendinopathy in flexor tendons, and females are 6 times more likely to be affected compared with males $(33,34)$. In addition, a previous study demonstrated that estrogen exhibits a protective effect on the tendon extracellular matrix, in particular collagen synthesis in females (35). Therefore, in the present study, to evaluate the aging-associated changes in tendons, postmenopausal female mice ( 78 weeks of age) were utilized. It should be clarified in future studies whether the changes observed in aged female mice are also present in aged male mice. Additionally, it has been suggested that treatment with phytoestrogens and exercise loading are effective for the maintenance of tendon mass and mechanical properties $(35,36)$. Therefore, it would be important to determine whether phytoestrogens and exercise suppress and exhibit protective actions on the aging-associated decrease in the expression of collagen and tenogenic markers observed in the present study.

The present study evaluated the changes in tendon structure and expression of tendon-associated genes accompanied with aging, using flexor tendons in a mouse model. The results demonstrated that the structure of the tendons, distribution of types I and III collagen and the expression of tendon-associated genes were modulated by aging in the flexor tendon, and these changes may contribute to the degeneration of tendons in tendinopathy. We hypothesize that the factors examined in the 
present study, including the structure of the tendons, distribution of types I and III collagen and the expression of tendon-associated genes, may be utilized in the future for the assessment of the protective actions of treatment with estrogen-associated substances (phytoestrogens) and exercise on the tendinopathy, including tenosynovitis and tendon rupture.

\section{Acknowledgements}

The authors would like to thank Dr Takako Ikegami (Research Support Center, Juntendo University Graduate School of Medicine, Tokyo, Japan) for their technical expertise in the RT-qPCR protocols.

\section{Funding}

No funding was received.

\section{Availability of data and materials}

The datasets used and/or analyzed during the present study are available from the corresponding author on reasonable request.

\section{Authors' contributions}

YS, KG and KN designed the study; KK, YS, YK, AF, MI and IN analyzed and interpreted the data regarding histochemical staining and gene expression; YS and IN were major contributors in writing the manuscript. All authors read and approved the final manuscript.

\section{Ethics approval and consent to participate}

The present study was approved by the Animal Care Committee of Juntendo University (registration no. 1309; approval no. 300052).

\section{Patient consent for publication}

Not applicable.

\section{Competing interests}

The authors declare that they have no competing interests.

\section{References}

1. Bhatia-Dey N, Kanherkar RR, Stair SE, Makarev EO and Csoka AB: Cellular Senescence as the Causal Nexus of Aging. Front Genet 7: 13, 2016.

2. López-Otín C, Blasco MA, Partridge L, Serrano M and Kroemer G: The hallmarks of aging. Cell 153: 1194-1217, 2013.

3. Drake MT, Clarke BL and Lewiecki EM: The Pathophysiology and Treatment of Osteoporosis. Clin Ther 37: 1837-1850, 2015.

4. Carmeli E, Patish $\mathrm{H}$ and Coleman R: The aging hand. J Gerontol A Biol Sci Med Sci 58: 146-152, 2003.

5. Quan T and Fisher GJ: Role of Age-Associated Alterations of the Dermal Extracellular Matrix Microenvironment in Human Skin Aging: A Mini-Review. Gerontology 61: 427-434, 2015.

6. Andarawis-Puri N, Flatow EL and Soslowsky LJ: Tendon basic science: Development, repair, regeneration, and healing. J Orthop Res 33: 780-784, 2015.

7. Cook JL and Purdam C: Is compressive load a factor in the development of tendinopathy? Br J Sports Med 46: 163-168, 2012.
8. Wu YT, Wu PT and Jou IM: Peritendinous elastase treatment induces tendon degeneration in rats: A potential model of tendinopathy in vivo. J Orthop Res 34: 471-477, 2016.

9. Ackerman JE, Bah I, Jonason JH, Buckley MR and Loiselle AE: Aging does not alter tendon mechanical properties during homeostasis, but does impair flexor tendon healing. J Orthop Res 35: 2716-2724, 2017.

10. Kostrominova TY and Brooks SV: Age-related changes in structure and extracellular matrix protein expression levels in rat tendons. Age (Dordr) 35: 2203-2214, 2013.

11. Loppini M, Longo UG, Niccoli G, Khan WS, Maffulli N and Denaro V: Histopathological scores for tissue-engineered, repaired and degenerated tendon: A systematic review of the literature. Curr Stem Cell Res Ther 10: 43-55, 2015.

12. Soslowsky LJ, Carpenter JE, DeBano CM,Banerji I and Moalli MR: Development and use of an animal model for investigations on rotator cuff disease. J Shoulder Elbow Surg 5: 383-392, 1996.

13. Borges LF, Gutierrez PS, Marana HR and Taboga SR: Picrosirius-polarization staining method as an efficient histopathological tool for collagenolysis detection in vesical prolapse lesions. Micron 38: 580-583, 2007.

14. Junqueira LC, Cossermelli W and Brentani R: Differential staining of collagens type I, II and III by Sirius Red and polarization microscopy. Arch Histol Jpn 41: 267-274, 1978.

15. Zerbinati $\mathrm{N}$ and Calligaro $\mathrm{A}$ : Calcium hydroxylapatite treatment of human skin: Evidence of collagen turnover through picrosirius red staining and circularly polarized microscopy. Clin Cosmet Investig Dermatol 11: 29-35, 2018.

16. Geary MB, Orner CA, Bawany F, Awad HA, Hammert WC, O'Keefe RJ and Loiselle AE: Systemic EP4 Inhibition Increases Adhesion Formation in a Murine Model of Flexor Tendon Repair. PLoS One 10: e0136351, 2015.

17. Livak KJ and Schmittgen TD: Analysis of relative gene expression data using real-time quantitative PCR and the $2(-\Delta \Delta$ C(T)) method. Methods 25: 402-408, 2001.

18. Maffulli N, Barrass V and Ewen SW: Light microscopic histology of achilles tendon ruptures. A comparison with unruptured tendons. Am J Sports Med 28: 857-863, 2000.

19. Thorpe CT, Streeter I, Pinchbeck GL, Goodship AE, Clegg PD and Birch HL: Aspartic acid racemization and collagen degradation markers reveal an accumulation of damage in tendon collagen that is enhanced with aging. J Biol Chem 285: 15674-15681, 2010.

20. Wang JH: Mechanobiology of tendon. J Biomech 39: 1563-1582, 2006.

21. Duance VC, Restall DJ, Beard H, Bourne FJ and Bailey AJ: The location of three collagen types in skeletal muscle. FEBS Lett 79: 248-252, 1977.

22. Suzuki H, Ito $Y$, Shinohara M, Yamashita S, Ichinose $S$, Kishida A, Oyaizu T, Kayama T, Nakamichi R, Koda N, et al: Gene targeting of the transcription factor Mohawk in rats causes heterotopic ossification of Achilles tendon via failed tenogenesis. Proc Natl Acad Sci USA 113: 7840-7845, 2016.

23. Murchison ND, Price BA, Conner DA, Keene DR, Olson EN, Tabin CJ and Schweitzer R: Regulation of tendon differentiation by scleraxis distinguishes force-transmitting tendons from muscle-anchoring tendons. Development 134: 2697-2708, 2007.

24. Docheva D, Hunziker EB, Fässler R and Brandau O: Tenomodulin is necessary for tenocyte proliferation and tendon maturation. Mol Cell Biol 25: 699-705, 2005.

25. Oshiro W, Lou J, Xing X, Tu Y and Manske PR: Flexor tendon healing in the rat: A histologic and gene expression study. J Hand Surg Am 28: 814-823, 2003.

26. Kjaer M, Magnusson P, Krogsgaard M, Boysen Møller J, Olesen J, Heinemeier K, Hansen M, Haraldsson B, Koskinen S, Esmarck B, et al: Extracellular matrix adaptation of tendon and skeletal muscle to exercise. J Anat 208: 445-450, 2006.

27. Klatte-Schulz F, Pauly S, Scheibel M, Greiner S, Gerhardt C, Schmidmaier G and Wildemann B: Influence of age on the cell biological characteristics and the stimulation potential of male human tenocyte-like cells. Eur Cell Mater 24: 74-89, 2012.

28. Kohler J, Popov C, Klotz B, Alberton P, Prall WC, Haasters F, Müller-Deubert S, Ebert R, Klein-Hitpass L, Jakob F, et al: Uncovering the cellular and molecular changes in tendon stem/progenitor cells attributed to tendon aging and degeneration. Aging Cell 12: 988-999, 2013.

29. Zhou Z, Akinbiyi T, Xu L, Ramcharan M, Leong DJ, Ros SJ, Colvin AC, Schaffler MB, Majeska RJ, Flatow EL, et al: Tendon-derived stem/progenitor cell aging: Defective self-renewal and altered fate. Aging Cell 9: 911-915, 2010. 
30. Wang C, Rong YH, Ning FG and Zhang GA: The content and ratio of type I and III collagen in skin differ with age and injury. Afr J Biotechnol 10: 2524-2529, 2011.

31. Chen J, Wang A, Xu J and Zheng M: In chronic lateral epicondylitis, apoptosis and autophagic cell death occur in the extensor carpi radialis brevis tendon. J Shoulder Elbow Surg 19: 355-362, 2010.

32. Tokunaga T, Shukunami C, Okamoto N, Taniwaki T, Oka K, Sakamoto H, Ide J, Mizuta H and Hiraki Y: FGF-2 Stimulates the Growth of Tenogenic Progenitor Cells to Facilitate the Generation of Tenomodulin-Positive Tenocytes in a Rat Rotator Cuff Healing Model. Am J Sports Med 43: 2411-2422, 2015.

33. Adams JE and Habbu R: Tendinopathies of the Hand and Wrist. J Am Acad Orthop Surg 23: 741-750, 2015.

34. Miyamoto H, Miura T, Isayama H, Masuzaki R, Koike K and Ohe T: Stiffness of the first annular pulley in normal and trigger fingers. J Hand Surg Am 36: 1486-1491, 2011.
35. Ramos JE, Al-Nakkash L, Peterson A, Gump BS, Janjulia T, Moore MS, Broderick TL and Carroll CC: The soy isoflavone genistein inhibits the reduction in Achilles tendon collagen content induced by ovariectomy in rats. Scand J Med Sci Sports 22: e108-e114, 2012.

36. Thomopoulos S, Parks WC, Rifkin DB and Derwin KA Mechanisms of tendon injury and repair. J Orthop Res 33: $832-839,2015$. 